\title{
Out-of-equilibrium steady states of a locally driven lossy qubit array
}

\author{
Shovan Dutta $\odot^{*}$ and Nigel R. Cooper ${ }^{\dagger}$ \\ T.C.M. Group, Cavendish Laboratory, University of Cambridge, JJ Thomson Avenue, Cambridge CB3 OHE, United Kingdom
}

(Received 17 July 2020; accepted 12 January 2021; published 16 February 2021)

\begin{abstract}
We find a rich variety of counterintuitive features in the steady states of a qubit array coupled to a dissipative source and sink at two arbitrary sites, using a master equation approach. We show there are setups where increasing the pump and loss rates establishes long-range coherence. At sufficiently strong dissipation, the source or sink effectively generates correlation between its neighboring sites, leading to a striking density-wave order for a class of "resonant" geometries. This effect can be used more widely to engineer nonequilibrium phases. We show the steady states are generically distinct for hard-core bosons and free fermions, and differ significantly from the ones found before in special cases. They are explained by generally applicable ansatzes for the long-time dynamics at weak and strong dissipation. Our findings are relevant for existing photonic setups.
\end{abstract}

DOI: 10.1103/PhysRevResearch.3.L012016

\section{INTRODUCTION}

Environmental decoherence has long been seen as an unavoidable roadblock to stabilizing quantum phases for long periods of time [1]. However, rapid advances in cooling and trapping techniques over the last decades have led to experimental platforms where the coupling to the environment can be controlled and even engineered to an unprecedented degree [2]. As several studies have shown, such tailored dissipation can be used to prepare novel quantum states [3-5]. The competition between Hamiltonian dynamics and incoherent dissipation can produce feature-rich steady states with no analog in equilibrium condensed matter [6]. Understanding these nonequilibrium phases is of fundamental interest [7], with potential applications in quantum computing [8].

A prototypical experimental setup for exploring such states is a one-dimensional (1D) array of qubits coupled to local reservoirs. In particular, the qubits can be realized by hardcore bosons on a lattice [9] or, equivalently, a spin-1/2 XY chain, and the reservoir(s) can be designed to inject or remove a particle (or flip spin) at a given site, as in Ref. [10]. Theoretical studies modeling the resulting dynamics have focused almost exclusively on the cases where the pump and loss occur at opposite ends of the chain. Then the system can be reduced to free fermions [11], enabling special analytical approaches that have been used to examine nonequilibrium transport [11-17] and phase transitions [18-20]. However, without additional Zeeman fields, the steady state for end drives is rather featureless, with no long-range order [18,21]. On the other hand, a recent work showed that, for pump and

\footnotetext{
*sd843@cam.ac.uk

†nrc25@cam.ac.uk
}

loss at the center, there are multiple steady states with longrange coherence that are distinct from free fermions and arise from a dynamical symmetry [22]. Such disparate results raise the question of what happens for generic pump-loss configurations, not addressed by the end-driven [11] or center-driven [22] cases.

Here we characterize the steady states for generic setups with a single pump and a single loss site, finding several counterintuitive features which can be probed in already existing platforms [10]. In Sec. III, we use perturbation theory supported by numerics to show there are dipolelike arrangements where long-range coherence is induced by increasing dissipation. In Sec. IV, we show the steady state is generically nonthermal even at weak dissipation, contrary to what is known for symmetric setups [23]. Further, hard-core bosons and free fermions can form qualitatively distinct steady-state correlations, although their density profiles are always reflection symmetric. These attributes are explained by a simple product ansatz of the single-particle modes. In Sec. V, we find that at strong dissipation the chain is generally divided into a filled and an empty segment separated by a highentropy bulk. These segments are coupled by the source or sink which effectively produces correlation. Whenever two modes in neighboring segments come into resonance, this effect leads to striking density waves and long-range order. This is a geometric effect and can be generalized to multiple sources and sinks. We explain the oscillations by a modified ansatz, finding they are more robust in free fermions than in hard-core bosons.

These results highlight surprising phenomena that can arise in open many-body settings, elucidating differences between hard-core bosons and free fermions in 1D [24]. Our ansatzes apply to more general forms of dissipation, and reduce the numerical cost to linear in system size.

\section{MODEL AND KNOWN SPECIAL CASES}

We consider strongly interacting bosons on a 1D lattice in the hard-core limit [9], described by the 
Hamiltonian

$$
\hat{H}=-\hbar J \sum_{i=1}^{L-1}\left(\hat{b}_{i}^{\dagger} \hat{b}_{i+1}+\hat{b}_{i+1}^{\dagger} \hat{b}_{i}\right),
$$

where $\hat{b}_{i}^{\dagger}$ is the boson creation operator, $J$ is the hopping amplitude, and $L$ is the number of sites. The hard-core constraint is encoded in the relation $\hat{b}_{i}^{\dagger 2}=0$, which means no two bosons can occupy the same site. This leads to the commutation rules $\left[\hat{b}_{i}, \hat{b}_{j}\right]=0$ and $\left[\hat{b}_{i}, \hat{b}_{j}^{\dagger}\right]=(-1)^{\hat{n}_{i}} \delta_{i j}$, where $\hat{n}_{i}:=\hat{b}_{i}^{\dagger} \hat{b}_{i}$ is the local occupation. Such a system is equivalent to a spin-1/2 $X X$ chain, and has been realized with cold atoms in optical lattices [25-27] and microwave photons in nonlinear resonators [10]. The Hamiltonian is reduced to free fermions by the Jordan-Wigner map

$$
\hat{f}_{j}=(-1)^{\sum_{i<j} \hat{n}_{i}} \hat{b}_{j},
$$

where $\hat{f}_{j}$ are the free fermion operators. The transformed Hamiltonian simply reads $\hat{H}=-\hbar J \sum_{i}\left(\hat{f}_{i}^{\dagger} \hat{f}_{i+1}+\right.$ H.c. $)$.

The system of bosons is coupled to bosonic reservoirs that inject particles at a site $p$, if it is empty, and remove bosons from a site $q$, if it is occupied. Such sources and sinks can be engineered using transmon qubits in microwave circuits [10] and local addressing in optical traps [28-30]. We assume the reservoirs are Markovian, i.e., they relax much faster than the system-reservoir coupling, which is standard for these setups [31]. Upon tracing out the environment, the density operator $\hat{\rho}$ is governed by the master equation [7,31-34]

$$
\frac{d \hat{\rho}}{d t}=\mathcal{L} \hat{\rho}:=-\frac{i}{\hbar}[\hat{H}, \hat{\rho}]+\sum_{\alpha} \hat{L}_{\alpha} \hat{\rho} \hat{L}_{\alpha}^{\dagger}-\frac{1}{2}\left\{\hat{L}_{\alpha}^{\dagger} \hat{L}_{\alpha}, \hat{\rho}\right\},
$$

where we have two Lindblad operators modeling the dissipation, $\hat{L}_{+}:=\sqrt{\gamma_{+}} \hat{b}_{p}^{\dagger}$ and $\hat{L}_{-}:=\sqrt{\gamma_{-}} \hat{b}_{q}, \gamma_{ \pm}$being the pump and loss rates, respectively. Note that Eq. (2) does not, in general, reduce the full dynamics to free fermions with (local) pump and loss. Instead, the dissipation mediates nonlocal interactions between the fermions.

The above system has been studied most widely when the source and sink are at opposite ends, i.e., $p=1$ and $q=L$. Then the only term in Eq. (3) that differs from the freefermion case is $\hat{L}_{-} \hat{\rho} \hat{L}_{-}^{\dagger}=\gamma_{-} \hat{f_{L}} \hat{P} \hat{\rho} \hat{P} \hat{f}_{L}^{\dagger}$, where $\hat{P}$ is the total particle-number parity. Since $\hat{P}$ is conserved by the Hamiltonian, one can show the dynamics decouple into sectors with $\hat{P} \hat{\rho} \hat{P}= \pm \hat{\rho}$. Thus, the Liouvillian $\mathcal{L}$ becomes quadratic in the free fermions. In such cases, the full solution can be found from the spectral properties of a $4 L \times 4 L$ matrix using quantization in the space of operators [11]. The steady state is identical to that of end-driven free fermions, characterized by a uniform bulk with short-range correlations [21], as shown in Fig. 1(a). We present a closed-form analytic solution for this end-driven case in the Supplemental Material [35].

The end-driven case is in sharp contrast to the scenario where pump and loss both occur at the center site (for odd $L$ ), which we explored in a recent work [22]. Here, the system does not map onto free fermions. Instead, one has multiple steady states due to a symmetry operator $\hat{C}=-1 / 2+$ $\sum_{k} \hat{f}_{L+1-k}^{\dagger} \hat{f}_{k}$, which splits the dynamics into $(L+1) / 2$ sectors with varying degrees of entanglement. The symmetry stabilizes particle-hole pairs at reflection-symmetric sites $k$ and $\tilde{k}:=L+1-k$, leading to steady states with long-range

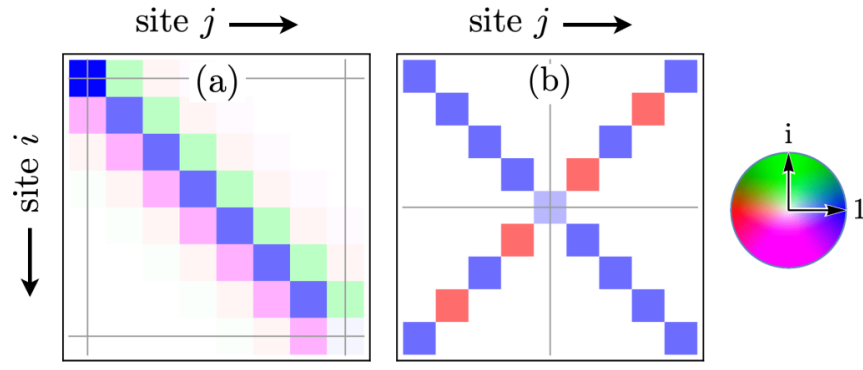

FIG. 1. Contrasting steady-state correlations $\left\langle\hat{b}_{i}^{\dagger} \hat{b}_{j}\right\rangle$ of hard-core bosons on a 1D lattice subject to incoherent pump with rate $\gamma_{+}$and loss with rate $\gamma_{-}$(a) at opposite ends, with $\gamma_{+}=\gamma_{-}=10 \mathrm{~J}$, and (b) at the center, with $\gamma_{-}=4 \gamma_{+}$, where $J$ is the tunneling.

coherence, as shown in Fig. 1(b) for the maximally entangled sector. In comparison, center-driven free fermions have an exponentially large set of steady states, as all odd single-particle wave functions vanish at the center.

Outside the above two scenarios, it is known that driving with both pump and loss at a generic site (not center) yields the product state $\hat{\rho}_{0} \propto \otimes_{i}\left(\gamma_{+}\left|1_{i}\right\rangle\left\langle 1_{i}\left|+\gamma_{-}\right| 0_{i}\right\rangle\left\langle 0_{i}\right|\right)$ [36], which is an infinite-temperature state with chemical potential $\mu=$ $\ln \left(\gamma_{+} / \gamma_{-}\right)$. We find free fermions also have the same steady state, except when a single-particle state vanishes at the drive site, producing degeneracies.

\section{DISSIPATION-INDUCED LONG-RANGE COHERENCE}

As described above, long-range order is absent for end drives, and restored for center drives by a special symmetry, irrespective of the pump/loss rate [22]. Here we find examples where long-range coherence is established by increasing dissipation. In particular, consider a "dipole" setup where pump and loss occur at two neighboring sites in the middle, i.e., $p=L / 2$ and $q=L / 2+1$, for even $L$. This can be seen as a center-drive analog, but there is no strong symmetry [37] and the steady state is unique. Using first-order perturbation theory at weak dissipation $\left(\gamma_{ \pm} \ll J\right)$, we find the steady state (see Supplemental Material [35])

$$
\hat{\rho}_{\mathrm{w}} \approx\left[1+i \frac{\gamma_{+}+\gamma_{-}}{2 J}\left(\hat{Q}-\hat{Q}^{\dagger}\right)\right] \hat{\rho}_{0},
$$

where $\hat{Q}:=\sum_{k=1}^{L / 2} \hat{f}_{\tilde{k}}^{\dagger} \hat{f}_{k}$, and $\hat{\rho}_{0}$ is the product state with uniform occupation $n_{0}=\gamma_{+} /\left(\gamma_{+}+\gamma_{-}\right)$. The perturbation $\hat{Q}$ is reminiscent of the symmetry operator $\hat{C}$, and generates the antidiagonal correlations

$$
\left\langle\hat{b}_{k}^{\dagger} \hat{b}_{\tilde{k}}\right\rangle_{\mathrm{w}} \approx i \frac{\gamma_{+} \gamma_{-}}{2 J\left(\gamma_{+}+\gamma_{-}\right)}\left(\frac{\gamma_{+}-\gamma_{-}}{\gamma_{+}+\gamma_{-}}\right)^{L-2 k} .
$$

Thus, at weak dissipation, the coherences decay exponentially with distance, and are limited to nearest neighbors $(k=L / 2)$ for $\gamma_{+}=\gamma_{-}$, as shown in Fig. 2(a). Conversely, for strong dissipation, the steady state approaches $\hat{\rho}_{\text {step }}$ where all sites $i \leqslant L / 2$ are filled and $i>L / 2$ are empty. To first order in $J / \gamma_{ \pm}$, we find (see Supplemental Material [35]),

$$
\hat{\rho}_{\mathrm{s}} \approx \hat{\rho}_{\text {step }}+i \frac{2 J}{\gamma_{+}+\gamma_{-}}\left(\hat{Q} \hat{\rho}_{\text {step }}-\text { H.c. }\right)
$$



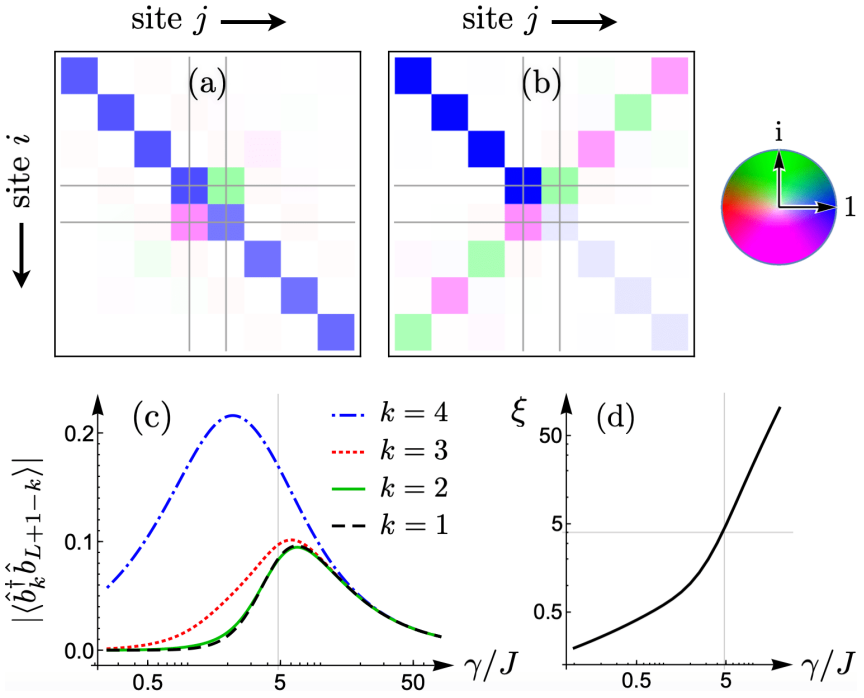

FIG. 2. Steady-state correlations $\left\langle\hat{b}_{i}^{\dagger} \hat{b}_{j}\right\rangle$ for pump and loss at neighboring sites $p=L / 2, q=L / 2+1$ for $\gamma_{+}=\gamma_{-}:=\gamma$ and $L=$ 8 at (a) weak dissipation, $\gamma=0.6 J$, and (b) strong dissipation, $\gamma=$ $8 J$. (c) Coherence between reflection-symmetric sites as a function of the pump/loss rate. (d) Correlation length $\xi$ obtained by fitting the coherences to an exponential.

and

$$
\left\langle\hat{b}_{k}^{\dagger} \hat{b}_{\tilde{k}}\right\rangle_{\mathrm{s}} \approx i \frac{2 J}{\gamma_{+}+\gamma_{-}}(-1)^{L / 2-k} .
$$

As shown in Fig. 2(b), now the coherences span the entire system, similar to the center-driven case [Fig. 1(b)]. These results imply that long-range coherence is induced by increasing the pump/loss relative to tunneling, which is confirmed by exact numerics [Fig. 2(c) and 2(d)]. The correlation length grows monotonically with dissipation, exceeding the system size for $\gamma_{ \pm} \gtrsim 5 J$. Note the perturbative expansion at strong dissipation is independent of the system size, so for any given $L$, the system approaches a unique steady state with long-range coherence. In contrast, for end drives [Fig. 1(a)], coherences are limited to nearest neighbors at both weak and strong dissipation, yielding (see Supplemental Material [35])

$$
\left\langle\hat{b}_{j}^{\dagger} \hat{b}_{j+1}\right\rangle_{\mathrm{w}} \approx i \frac{\gamma_{+} \gamma_{-}}{2 J\left(\gamma_{+}+\gamma_{-}\right)} ; \quad\left\langle\hat{b}_{j}^{\dagger} \hat{b}_{j+1}\right\rangle_{\mathrm{s}} \approx i \frac{2 J}{\gamma_{+}+\gamma_{-}} .
$$

Numerically, we find similar results in "dipole" setups with $q=p+1$, whenever $p$ divides $L-p$ or vice versa (see Supplemental Material [35] for examples). In Sec. V, we discuss more general scenarios where such geometric resonances stabilize long-range order in the Zeno limit.

\section{NONTHERMAL STEADY STATES AT WEAK DISSIPATION}

For end drives as well as for the "dipole" setup considered in Sec. III, the steady state approaches the uniform infinitetemperature state $\hat{\rho}_{0}$ in the weak-dissipation limit. Here, the system has time to equilibrate between successive pump and loss events. Thus, one might expect the same steady state regardless of where those events occur. This is indeed true whenever the pump and loss act on reflection-symmetric sites
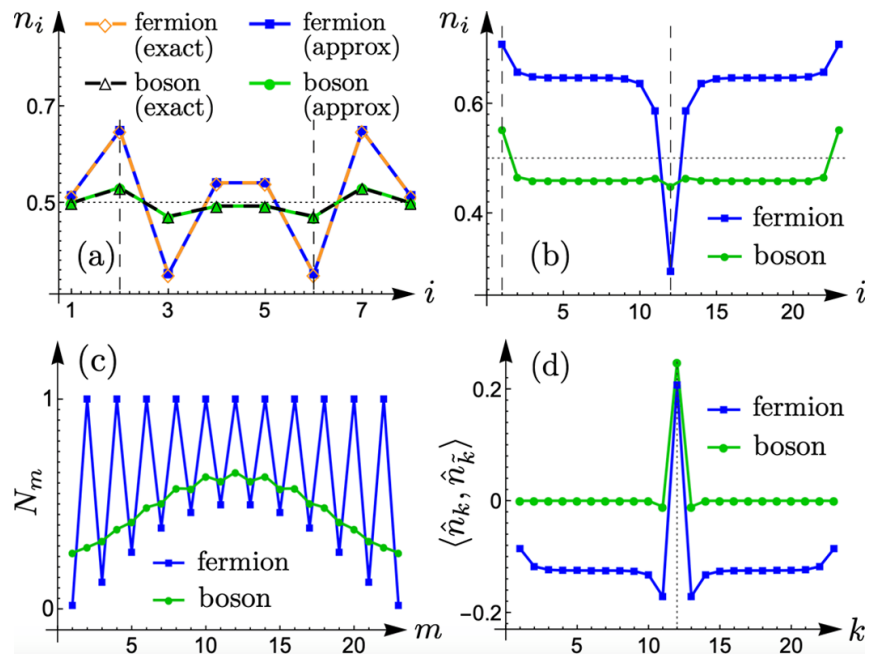

FIG. 3. Steady-state density $n_{i}$ of free fermions and hard-core bosons in the weak-dissipation limit with $\gamma_{+}=\gamma_{-}$for (a) $L=8$, $p=2, q=6$, from exact diagonalization and using a product-ofmodes ansatz [Eqs. (10)-(11)], and (b) $L=23, p=1, q=12$, using the ansatz. (c) Occupation of single-particle modes and (d) density correlation between sites $k$ and $\tilde{k}:=L+1-k$ for the setup in (b), where $\left\langle\hat{n}_{i}, \hat{n}_{j}\right\rangle:=\left\langle\hat{n}_{i} \hat{n}_{j}\right\rangle-n_{i} n_{j}$.

[23] or at the same site (except center) [36]. However, we find those are the only setups where the conjecture holds. As shown in Figs. 3(a)-3(b), the steady state is generically nonthermal, and different for free fermions and hard-core bosons, although they both have symmetric densities. These features can be understood by focusing instead on the single-particle modes, which are unaffected by the Hamiltonian. They are given by unitary maps $\hat{F}_{m}=\sum_{j} c_{m, j} \hat{f}_{j}$ where

$$
c_{m, j}=\sqrt{2 /(L+1)} \sin [\pi m j /(L+1)],
$$

and have energy $\varepsilon_{m}=-2 \hbar J \cos \left(\frac{\pi m}{L+1}\right), m=1, \ldots, L$. If the dissipation is small compared to the energy splitting, the modes become uncorrelated. Thus, we find the steady state is well approximated by a "product-of-modes" form $\hat{\rho} \approx \prod_{m}\left(1-N_{m}\right)\left|0_{m}\right\rangle\left\langle 0_{m}\left|+N_{m}\right| 1_{m}\right\rangle\left\langle 1_{m}\right|$, set by the mode occupations $N_{m}$. The lack of correlation explains why the density is symmetric. Using $\left\langle\hat{F}_{m}^{\dagger} \hat{F}_{n}\right\rangle \approx \delta_{m n} N_{m}$ yields $n_{j}:=\left\langle\hat{n}_{j}\right\rangle=\sum_{m} N_{m}\left|c_{m, j}\right|^{2}$, which gives $n_{\tilde{j}}=n_{j}$ for any site $j$. (Recall that $\tilde{j}:=L+1-j$ ).

One can use the product-of-modes ansatz in Eq. (3) to construct approximate rate equations for $N_{m}$. For free fermions, one finds (see Supplemental Material [35])

$$
\dot{N}_{m} \approx \gamma_{+}\left|c_{m, p}\right|^{2}\left(1-N_{m}\right)-\gamma_{-}\left|c_{m, q}\right|^{2} N_{m}
$$

i.e., the modes are uncoupled, with incoming and outgoing currents set by the weight of the respective mode at the pump and loss sites. To reach a uniform steady state such as $\hat{\rho}_{0}$, one must have $\left|c_{m, p}\right|=\left|c_{m, q}\right| \forall m$, which is satisfied iff $q=p$ or $q=\tilde{p}$. While this conclusion also holds for hard-core bosons, the rate equations are more complex as the string operator in Eq. (2) generates nonlinear coupling between the modes. Using $\left\langle\hat{F}_{m}^{\dagger} \hat{F}_{m^{\prime}}^{\dagger} \hat{F}_{n} \hat{F}_{n^{\prime}}\right\rangle \approx N_{n} N_{n^{\prime}}\left(\delta_{m, n^{\prime}} \delta_{m^{\prime}, n}-\delta_{m, n} \delta_{m^{\prime}, n^{\prime}}\right)$, we find 
(see derivation in the Supplemental Material [35])

$$
\begin{aligned}
\dot{N}_{m} \approx & \gamma_{+}\left[\bar{N}_{m}\left(N_{m}\left|c_{m, p}\right|^{2}+\bar{n}_{p}\right)-\bar{n}_{p} \bar{\beta}_{m}^{(p)}+\left|\kappa_{m}^{(p)}-c_{m, p}\right|^{2}\right] \\
& -\gamma_{-}\left[N_{m}\left(\bar{N}_{m}\left|c_{m, q}\right|^{2}+n_{q}\right)-n_{q} \beta_{m}^{(q)}+\left|\kappa_{m}^{(q)}\right|^{2}\right],
\end{aligned}
$$

with notation $\quad \bar{x}:=1-x, \quad \beta_{m}^{(i)}:=\sum_{n} N_{n}\left|\alpha_{m, n}^{(i)}\right|^{2}, \quad \kappa_{m}^{(i)}:=$ $\sum_{n} N_{n} \alpha_{n, m}^{(i)} c_{n, i}$, where $\alpha_{m, n}^{(i)}:=\left(\sum_{j \geqslant i}-\sum_{j<i}\right) c_{m, j}^{*} c_{n, j}$. In numerical trials, Eq. (11) gives a unique steady state with $0 \leqslant N_{m} \leqslant 1$ for all $m$.

Figure 3(a) shows the ansatz accurately describes the steady states in the weak-dissipation limit, becoming exact for free fermions. The densities are peaked at the pump site and minimized at the loss site, with $n_{q}=1-n_{p}$ for $\gamma_{+}=\gamma_{-}$. The same is mirrored at sites $\tilde{p}$ and $\tilde{q}$, which explains why choosing $q=\tilde{p}$ [23] or $q=p$ [36] gives half filling at all sites. In general, the density fluctuations are significantly smaller for hard-core bosons due to strong interactions. There are also qualitative differences which persist to large system sizes, as shown in Figs. 3(b)-3(d). Here one has pump at one end and loss at the center. For free fermions, all the odd modes (even $m$ ) are immune to loss and thus fully filled, which is not the case for hard-core bosons [Fig. 3(c)]. Thus, we find strikingly different densities and correlations. In particular, Fig. 3(d) shows that unlike bosons, fermions exhibit long-range order in the density correlations $\left\langle\hat{n}_{i} \hat{n}_{j}\right\rangle-n_{i} n_{j} \approx \delta_{i j} n_{i}-\left|\left\langle\hat{f}_{i}^{\dagger} \hat{f}_{j}\right\rangle\right|^{2}$ [35], which holds more generally at weak dissipation. Also, note the free fermions have degenerate steady states if any of the modes vanishes at both pump and loss sites, whereas for hard-core bosons the steady state is unique except for center drive [22]. Equations (10) and (11) apply for any free-fermionic Hamiltonian with nondegenerate spectrum, reducing the dynamics to $L$ rate equations.

\section{GEOMETRIC RESONANCE AND LONG-RANGE ORDER IN THE ZENO LIMIT}

In Sec. III we found, for pump and loss at neighboring sites, $q=p+1$, the steady state at strong dissipation approaches a step where sites 1 through $p$ are filled and sites $q$ through $L$ are empty [Eq. (6)]. This can be understood as follows. For $t \gtrsim 1 / \gamma_{ \pm}$, sites $p$ and $q$ are pinned at occupation 1 and 0 , respectively. By tracing over this subspace, one can show the remaining sites are governed by a master equation with an effective Hamiltonian $\hat{H}_{\text {eff }}$ and weak effective dissipation, as detailed in Ref. [38] for more general systems. In our setup, $\hat{H}_{\text {eff }}$ simply describes hopping in the disjoint segments 1 to $p-1, p+1$ to $q-1$, and $q+1$ to $L$. For $q=p+1$, the middle region is absent and the dissipation is given by Lindblad operators $\hat{L}_{+}^{\text {eff }}=\sqrt{\Gamma_{+}} \hat{b}_{p-1}^{\dagger}$ and $\hat{L}_{-}^{\text {eff }}=\sqrt{\Gamma_{-}} \hat{b}_{q+1}$ where $\Gamma_{ \pm}:=$ $4 J^{2} / \gamma_{ \pm}$(see Supplemental Material [35]). The former injects particles into the first segment until it is filled, and the latter removes all particles from the last segment. More generally,

$$
\hat{L}_{+}^{\text {eff }}=\sqrt{\Gamma_{+}}\left(\hat{b}_{p-1}^{\dagger}+\hat{b}_{p+1}^{\dagger}\right), \quad \hat{L}_{-}^{\text {eff }}=\sqrt{\Gamma_{-}}\left(\hat{b}_{q-1}+\hat{b}_{q+1}\right),
$$

i.e., the source and sink induce correlated pump and loss at neighboring sites with rate $\Gamma_{ \pm}$, coupling the segments. However, this is a second-order effect $\left(\Gamma_{ \pm} / J \sim J^{2} / \gamma_{ \pm}^{2}\right)$, and the steady state remains uncorrelated whenever the energy splitting between modes in adjacent segments is large compared to $\Gamma_{ \pm}$, as we explain below. Then the mid region behaves like (a)


FIG. 4. (a) Resonant modes in adjacent segments for a specific pump-loss setup in the Zeno limit, $\gamma_{ \pm} \gg J$. (b) Steady-state correlation between free-fermion modes in segments $v=1,2,3$, showing coherence among resonant modes. (c) Density of free fermions and hard-core bosons from exact diagonalization $\left(\gamma_{+}=\gamma_{-}\right)$.

an end-driven qubit array, reaching a uniform product state with density $n^{(2)}=\Gamma_{+} /\left(\Gamma_{+}+\Gamma_{-}\right)$. This is similar to $\hat{\rho}_{0}$ in Eq. (4) except $n^{(2)}$ decreases with $\gamma_{+} / \gamma_{-}$, a consequence of the Zeno effect $[39,40]$. Thus, the steady state generically consists of fully filled and empty sites separated by a region of high entropy.

This picture breaks down if any two modes in neighboring segments are resonant. Then they can remain coherent via the correlated pump and loss, producing characteristic density waves as in Fig. 4. To understand this feature, note the singleparticle modes in segment $v$ are given by $\hat{F}_{m_{v}}^{(v)}=\sum_{j_{v}} c_{m_{v}, j_{v}}^{(v)}{\hat{j_{v}}}_{j_{v}}$, with amplitudes $c_{m_{v}, j_{v}}^{(\nu)}$ as in Eq. (9), for $m_{v}=1, \ldots, L_{v}$, where $L_{v}$ is the number of sites. They have energies $\varepsilon_{m_{v}}^{(v)}=-2 \hbar J \cos k_{m_{v}}^{(v)}$, where $k_{m_{v}}^{(v)}:=\pi m_{v} /\left(L_{v}+1\right)$. Thus, offresonant modes dephase at a rate $\Delta \varepsilon / \hbar \sim J$, much faster than the dissipative coupling $\Gamma_{ \pm}$. As a result, for $t \gtrsim 1 / \Gamma_{ \pm}$, adiabatic elimination gives $\left\langle\hat{F}_{m_{v}}^{(v) \dagger} \hat{F}_{m_{v+1}}^{(v+1)}\right\rangle \approx \Lambda_{m_{v}, m_{v+1}}^{(v, v+1)} \widetilde{T}_{m_{v}, m_{v+1}}^{(v, v+1)}$, where $\Lambda_{m_{v}, m_{v^{\prime}}}^{\left(v, v^{\prime}\right)}$ is 1 for resonant modes and 0 otherwise. Since the energies are set by the wave number $k_{m_{v}}^{(v)}$, the resonance condition is equivalent to a single plane wave fitting into both segments $v$ and $v+1$, as in Fig. 4(a). The corresponding modes are seen to have nonzero steady-state correlation in Fig. 4(b). Such modes exist iff $L_{v}+1$ divides $L_{v+1}+1$, or vice versa, making these arrangements special. Within each segment, one finds $\left\langle\hat{F}_{m_{v}}^{(v) \dagger} \hat{F}_{n_{v}}^{(v)}\right\rangle \approx \delta_{m_{v}, n_{v}} N_{m_{v}}^{(v)}$, as in Sec. IV, where $N_{m_{v}}^{(\nu)}$ are the mode occupations. These occupations are altered by the resonances, producing density waves in the two coupled segments [Fig. 4(c)], which are stronger in free fermions than in hard-core bosons.

The above attributes can be explained by approximate rate equations for the modes. For free fermions, one finds (see derivation in the Supplemental Material [35])

$$
\begin{aligned}
\dot{N}_{m_{3}}^{(3)} & \approx-\Gamma_{-}\left[\left|u_{m_{3}}^{(3)}\right|^{2} N_{m_{3}}^{(3)}+\operatorname{Re} \sum_{m_{2}} \Lambda_{m_{2}, m_{3}}^{(2,3)} v_{m_{2}}^{(2)} u_{m_{3}}^{(3) *} T_{m_{2}, m_{3}}^{(2,3)}\right], \\
\dot{T}_{m_{2}, m_{3}}^{(2,3)} & \approx-\Gamma_{f} T_{m_{2}, m_{3}}^{(2,3)}-\Gamma_{-} v_{m_{2}}^{(2) *} u_{m_{3}}^{(3)}\left[N_{m_{2}}^{(2)}+N_{m_{3}}^{(3)}\right] / 2,
\end{aligned}
$$

where $u_{m_{v}}^{(\nu)}:=c_{m_{\nu}, 1}^{(v)}$ and $v_{m_{v}}^{(v)}:=c_{m_{v}, L_{\nu}}^{(\nu)}$ are the mode amplitudes at the boundary where pump or loss occurs, and $\Gamma_{f}:=$ $\frac{\Gamma_{+}}{2}\left|u_{m_{2}}^{(2)}\right|^{2}+\frac{\Gamma_{-}}{2}\left[\left|v_{m_{2}}^{(2)}\right|^{2}+\left|u_{m_{3}}^{(3)}\right|^{2}\right]$ is an effective decay rate of the 
(a)

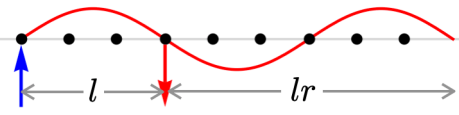

$n_{i}$
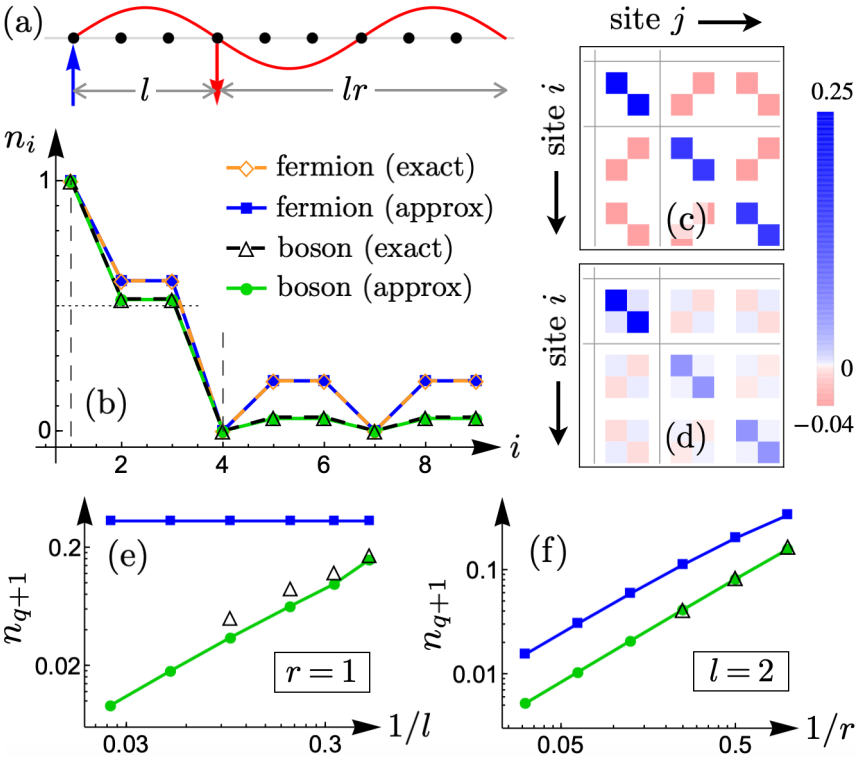

FIG. 5. (a) Lowest-energy resonant mode in a class of setups parametrized by integers $l=3$ and $r=2$. (b) Steady-state density of free fermions and hard-core bosons with $\gamma_{+}=\gamma_{-}$in the Zeno limit from exact diagonalization and our ansatz [Eq. (13)]. (c)-(d) Density-density correlations of the fermions and bosons, respectively. (e)-(f) Scaling of the density bump $n_{q+1}$ with system size, $q$ being the loss site, with the same color convention as in (b).

correlations. The equations for the remaining segments follow by symmetry [35]. Note the occupation $N_{m_{3}}^{(3)}$ decays to zero unless it is coupled with a resonant mode $m_{2}$, producing long-range density-density correlations [Fig. 5(c)]. The rate equations become exact in the limit $\gamma_{ \pm} / J \rightarrow \infty$. For hardcore bosons, Eq. (13) gains an interaction term $\left.\dot{T}_{m_{2}, m_{3}}^{(2,3)}\right|_{\text {int }}=$ $2\left\langle\hat{L}_{-}^{\mathrm{eff} \dagger} \hat{F}_{m_{2}}^{(2) \dagger} \hat{F}_{m_{3}}^{(3)} \hat{L}_{-}^{\mathrm{eff}}\right\rangle$, which can be approximated by pairwise contractions, as in Eq. (11). The main result is an increased decay rate, $\Gamma_{b}=\Gamma_{f}+2\left\langle\hat{L}_{-}^{\text {eff }} \hat{L}_{-}^{\text {eff }}\right\rangle$ (see Supplemental Material [35]), which weakens the correlations, making the resonant features less prominent.

To further elucidate the characteristic features of hard-core bosons and free fermions, we consider a set of pumploss configurations with $p=1, q=l+1$, and $L=(r+1) l$, for positive integers $l$ and $r$. Here the sink is positioned such that every $r$ th mode in the last segment is resonant with successive modes in the middle [Fig. 5(a)]. In steady state, this leads to a train of density bumps in the former, with nodes at every $l$ th site [Fig. 5(b)]. As expected from the preceding paragraph, the bumps are smaller for hardcore bosons, well reproduced by the resonant-modes ansatz. In addition, free fermions show long-range density correlations, similar to the center-driven case [22], repeating with period $2 l$ [Fig. 5(c)]. For hard-core bosons, these are less prominent and cloaked in a uniform background due to interactions [Fig. 5(d)]. The reduced density fluctuations for bosons originate from a faster decay of correlations. Using $c_{m_{2}, j_{2}}^{(2)} \sim 1 / \sqrt{l}, u_{m_{3}}^{(3)} \sim 1 / \sqrt{l r}$, and $N_{m_{2}}^{(2)} \sim O(1)$ in Eq. (13) for the resonant modes gives $\Gamma_{f} \sim O\left(\Gamma_{ \pm} / l\right)$ and $N_{m_{3}}^{(3)} \sim O(1)$ for free fermions, and $\Gamma_{b} \sim O\left(\Gamma_{ \pm}\right)$and $N_{m_{3}}^{(3)} \sim O(1 / l)$ for hard-core bosons. Thus, we find a density bump $n_{q+1} \approx \sum_{m_{3}}\left|u_{m_{3}}^{(3)}\right|^{2} N_{m_{3}}^{(3)} \sim 1 / L$ in the latter, whereas, in the former, $n_{q+1} \sim O(1 / r)$, regardless of $l$. This different scaling with system size is evident in Figs. 5(e) and 5(f). Since the physics is solely determined by geometry, the framework can readily incorporate multiple sources and sinks. Further, Eq. (13) and its bosonic counterpart remain valid in the presence of a trap as long as the spectrum in each segment is nondegenerate.

\section{SUMMARY AND OUTLOOK}

We have characterized a rich class of steady states that arise in a prototypical setting of two-level systems driven by localized pump and loss. For different arrangements, we find dissipation-induced long-range coherence (Fig. 2) and surprising geometric order at strong pump and loss (Fig. 5). These results could be observed by measuring local densities and density correlations in state-of-the-art photonic setups [41]. We have developed a general framework to approximate the long-time dynamics at weak and strong dissipation in terms of rate equations for the energy eigenmodes, which would be useful in other systems. Note the strongly dissipative limit is reached in practice for $\gamma_{ \pm} \gtrsim 10 \mathrm{~J}$. Quite generally, we find a local source or sink generates correlation between its neighboring sites [Eq. (12)], which can stabilize long-range order [22]. This effect is present whenever the lossy site equilibrates much faster $\left(\gamma_{ \pm}\right)$than the global relaxation rate $(J / L)$ [38], and provides strong motivation for using such dissipation to engineer correlated states of matter [42]. Thus, it would be valuable to extend our study to interacting Hamiltonians, such as an $X X Z$ chain [43] or a Hubbard model [7], and to higher dimensions [44] where there is no simple map between hard-core bosons and free fermions [45]. It would also be interesting to see how the effect is altered in the continuum. Note the local pump and loss drives current through an interacting medium. Thus, future work could harness such probes to extract useful information about the bulk [46] and investigate larger questions of nonequilibrium transport [47-49].

\section{ACKNOWLEDGMENTS}

This work was supported by EPSRC Grant No. EP/P009565/1 and by a Simons Investigator Award.
[1] M. Schlosshauer, Quantum decoherence, Phys. Rep. 831, 1 (2019).

[2] M. Müller, S. Diehl, G. Pupillo, and P. Zoller, Engineered open systems and quantum simulations with atoms and ions, Adv. At. Mol. Opt. Phys. 61, 1 (2012).
[3] S. Diehl, E. Rico, M. A. Baranov, and P. Zoller, Topology by dissipation in atomic quantum wires, Nat. Phys. 7, 971 (2011).

[4] Y. Lin, J. P. Gaebler, F. Reiter, T. R. Tan, R. Bowler, A. S. Sørensen, D. Leibfried, and D. J. Wineland, Dissipative 
production of a maximally entangled steady state of two quantum bits, Nature (London) 504, 415 (2013).

[5] A. W. Carr and M. Saffman, Preparation of Entangled and Antiferromagnetic States by Dissipative Rydberg Pumping, Phys. Rev. Lett. 111, 033607 (2013).

[6] L. M. Sieberer, M. Buchhold, and S. Diehl, Keldysh field theory for driven open quantum systems, Rep. Prog. Phys. 79, 096001 (2016).

[7] G. Kordas, D. Witthaut, P. Buonsante, A. Vezzani, R. Burioni, A. I. Karanikas, and S. Wimberger, The dissipative Bose-Hubbard model, Eur. Phys. J. Spec. Top. 224, 2127 (2015).

[8] F. Verstraete, M. M. Wolf, and J. I. Cirac, Quantum computation and quantum-state engineering driven by dissipation, Nat. Phys. 5, 633 (2009).

[9] M. A. Cazalilla, R. Citro, T. Giamarchi, E. Orignac, and M. Rigol, One dimensional bosons: From condensed matter systems to ultracold gases, Rev. Mod. Phys. 83, 1405 (2011).

[10] R. Ma, B. Saxberg, C. Owens, N. Leung, Y. Lu, J. Simon, and D. I. Schuster, A dissipatively stabilized Mott insulator of photons, Nature (London) 566, 51 (2019).

[11] T. Prosen, Third quantization: a general method to solve master equations for quadratic open Fermi systems, New J. Phys. 10, 043026 (2008).

[12] T. Prosen and M. Žnidarič, Matrix product simulations of nonequilibrium steady states of quantum spin chains, J. Stat. Mech. (2009) P02035.

[13] M. Žnidarič, Exact solution for a diffusive nonequilibrium steady state of an open quantum chain, J. Stat. Mech. (2010) L05002.

[14] M. Žnidarič, Spin Transport in a One-Dimensional Anisotropic Heisenberg Model, Phys. Rev. Lett. 106, 220601 (2011).

[15] T. Prosen, Open $X X Z$ Spin Chain: Nonequilibrium Steady State and a Strict Bound on Ballistic Transport, Phys. Rev. Lett. 106, 217206 (2011).

[16] M. Žnidarič, B. Žunkovič, and T. Prosen, Transport properties of a boundary-driven one-dimensional gas of spinless fermions, Phys. Rev. E 84, 051115 (2011).

[17] P. Kos and T. Prosen, Time-dependent correlation functions in open quadratic fermionic systems, J. Stat. Mech. (2017) 123103.

[18] T. Prosen and I. Pižorn, Quantum Phase Transition in a Farfrom-Equilibrium Steady State of an XY Spin Chain, Phys. Rev. Lett. 101, 105701 (2008).

[19] M. Žnidarič, Solvable quantum nonequilibrium model exhibiting a phase transition and a matrix product representation, Phys. Rev. E 83, 011108 (2011).

[20] L. Banchi, P. Giorda, and P. Zanardi, Quantum informationgeometry of dissipative quantum phase transitions, Phys. Rev. E 89, 022102 (2014)

[21] M. Žnidarič, A matrix product solution for a nonequilibrium steady state of an XX chain, J. Phys. A 43, 415004 (2010).

[22] S. Dutta and N. R. Cooper, Long-Range Coherence and Multiple Steady States in a Lossy Qubit Array, Phys. Rev. Lett. 125, 240404 (2020).

[23] B. Buča and T. Prosen, Exactly Solvable Counting Statistics in Open Weakly Coupled Interacting Spin Systems, Phys. Rev. Lett. 112, 067201 (2014).
[24] J. Yago Malo, E. P. L. van Nieuwenburg, M. H. Fischer, and A. J. Daley, Particle statistics and lossy dynamics of ultracold atoms in optical lattices, Phys. Rev. A 97, 053614 (2018).

[25] B. Paredes, A. Widera, V. Murg, O. Mandel, S. Fölling, I. Cirac, G. V. Shlyapnikov, T. W. Hänsch, and I. Bloch, TonksGirardeau gas of ultracold atoms in an optical lattice, Nature (London) 429, 277 (2004).

[26] T. Stöferle, H. Moritz, C. Schori, M. Köhl, and T. Esslinger, Transition from a Strongly Interacting 1D Superfluid to a Mott Insulator, Phys. Rev. Lett. 92, 130403 (2004).

[27] P. M. Preiss, R. Ma, M. E. Tai, A. Lukin, M. Rispoli, P. Zupancic, Y. Lahini, R. Islam, and M. Greiner, Strongly correlated quantum walks in optical lattices, Science 347, 1229 (2015).

[28] G. Barontini, R. Labouvie, F. Stubenrauch, A. Vogler, V. Guarrera, and H. Ott, Controlling the Dynamics of an Open Many-Body Quantum System with Localized Dissipation, Phys. Rev. Lett. 110, 035302 (2013).

[29] H. Schwager, J. I. Cirac, and G. Giedke, Dissipative spin chains: Implementation with cold atoms and steady-state properties, Phys. Rev. A 87, 022110 (2013).

[30] T. Fukuhara et al., Quantum dynamics of a mobile spin impurity, Nat. Phys. 9, 235 (2013).

[31] A. J. Daley, Quantum trajectories and open many-body quantum systems, Adv. Phys. 63, 77 (2014).

[32] G. Lindblad, On the generators of quantum dynamical semigroups, Commun. Math. Phys. 48, 119 (1976).

[33] V. Gorini, A. Kossakowski, and E. C. G. Sudarshan, Completely positive dynamical semigroups of $N$-level systems, J. Math. Phys. 17, 821 (1976).

[34] H.-P. Breuer and F. Petruccione, The Theory of Open Quantum Systems (Oxford University Press, Oxford, 2002).

[35] See Supplemental Material at http://link.aps.org/supplemental/ 10.1103/PhysRevResearch.3.L012016, which includes Refs. [[50-53]], for analytic and perturbative solution for steady states, derivation of effective dynamics and rate equations at weak and strong dissipation, and examples of dissipationinduced long-range coherence in resonant "dipole" geometries.

[36] I. Pižorn, One-dimensional Bose-Hubbard model far from equilibrium, Phys. Rev. A 88, 043635 (2013).

[37] B. Buča and T. Prosen, A note on symmetry reductions of the Lindblad equation: transport in constrained open spin chains, New J. Phys. 14, 073007 (2012).

[38] V. Popkov, S. Essink, C. Presilla, and G. Schütz, Effective quantum Zeno dynamics in dissipative quantum systems, Phys. Rev. A 98, 052110 (2018).

[39] B Misra and E. C. G. Sudarshan, The Zeno's paradox in quantum theory, J. Math. Phys. 18, 756 (1977).

[40] H. Fröml, C. Muckel, C. Kollath, A. Chiocchetta, and S. Diehl, Ultracold quantum wires with localized losses: Many-body quantum Zeno effect, Phys. Rev. B 101, 144301 (2020).

[41] I. Carusotto, A. A. Houck, A. J. Kollár, P. Roushan, D. I. Schuster, and J. Simon, Photonic materials in circuit quantum electrodynamics, Nat. Phys. 16, 268 (2020).

[42] V. Popkov, S. Essink, C. Kollath, and C. Presilla, Dissipative generation of pure steady states and a gambler's ruin problem, Phys. Rev. A 102, 032205 (2020).

[43] T. Prosen and M. Žnidarič, Long-Range Order in Nonequilibrium Interacting Quantum Spin Chains, Phys. Rev. Lett. 105, 060603 (2010). 
[44] M. F. Maghrebi and A. V. Gorshkov, Nonequilibrium manybody steady states via Keldysh formalism, Phys. Rev. B 93, 014307 (2016).

[45] E. Fradkin, Jordan-Wigner Transformation for Quantum-Spin Systems in Two Dimensions and Fractional Statistics, Phys. Rev. Lett. 63, 322 (1989).

[46] R. O. Umucalılar and I. Carusotto, Generation and spectroscopic signatures of a fractional quantum Hall liquid of photons in an incoherently pumped optical cavity, Phys. Rev. A 96, 053808 (2017).

[47] L. Corman, P. Fabritius, S. Häusler, J. Mohan, L. H. Dogra, D. Husmann, M. Lebrat, and T. Esslinger, Quantized conductance through a dissipative atomic point contact, Phys. Rev. A 100, 053605 (2019).

[48] S. Maity, S. Bandyopadhyay, S. Bhattacharjee, and A. Dutta, Growth of mutual information in a quenched one-dimensional open quantum many-body system, Phys. Rev. B 101, 180301(R) (2020).

[49] V. Alba and F. Carollo, Spreading of correlations in Markovian open quantum systems, Phys. Rev. B 103, L020302 (2021).

[50] T. Prosen, Comments on a boundary-driven open $X X Z$ chain: asymmetric driving and uniqueness of steady states, Phys. Scr. 86, 058511 (2012).

[51] H. Umezawa, Advanced Field Theory: Micro, Macro, and Thermal Physics (AIP, New York, 1993).

[52] M. V. Medvedyeva, F. H. L. Essler, and T. Prosen, Exact Bethe Ansatz Spectrum of a Tight-Binding Chain with Dephasing Noise, Phys. Rev. Lett. 117, 137202 (2016).

[53] D. Karevski, V. Popkov, and G. M. Schütz, Exact Matrix Product Solution for the Boundary-Driven Lindblad $X X Z$ Chain, Phys. Rev. Lett. 110, 047201 (2013). 